\title{
Self-assessment tool of disease activity of rheumatoid arthritis by using a smartphone application.
}

\section{$\operatorname{AUTHOR}(\mathrm{S}):$}

Nishiguchi, Shu; Ito, Hiromu; Yamada, Minoru; Yoshitomi, Hiroyuki; Furu, Moritoshi; Ito, Tatsuaki; Shinohara, Akio; Ura, Tetsuya; Okamoto, Kazuya; Aoyama, Tomoki

\section{CITATION:}

Nishiguchi, Shu ... [et al]. Self-assessment to ol of disease activity of rheumatoid arthritis by using a smartphone application.. Telemedicine journal and e-health : the official journal of the American Telemedicine Association 2014, 20(3): 235-240

\section{ISSUE DATE:}

2014-02-27

URL:

http://hdl.handle.net/2433/201380

\section{RIGHT:}

Final publication is available from Mary Ann Liebert, Inc., publishers

http://dx.doi.org/10.1089/tmj.2013.0162.; This is not the published version. Please cite only the published version.; この論文は出版社版でありません。引用の際には出版社版 をご確認ご利用ください。 
Self-assessment tool of disease activity of rheumatoid arthritis by using a smartphone application

\section{Running Title: Self-assessment tool of disease activity of RA}

\section{Authors}

Shu Nishiguchi, R.P.T., M.Sc. ${ }^{1,2 *}$, Hiromu Ito, M.D., Ph.D. ${ }^{3}$, Minoru Yamada, R.P.T., Ph.D. ${ }^{1}$, Hiroyuki Yoshitomi, M.D., Ph.D. ${ }^{4}$, Moritoshi Furu, M.D., Ph.D. ${ }^{3}$, Tatsuaki Ito, M.E. ${ }^{5}$, Akio Shinohara, M.E. ${ }^{5}$, Tetsuya Ura, M.E. ${ }^{5}$, Kazuya Okamoto, Ph.D. ${ }^{6}$, Tomoki Aoyama, M.D., Ph.D. ${ }^{1}$

\section{Affiliations}

1. Department of Physical Therapy, Human Health Sciences, Kyoto University Graduate School of Medicine, Kyoto, Japan

2. Japan Society for the Promotion of Science, Tokyo, Japan

3. Department of the Control for Rheumatic Diseases, Kyoto University Graduate School of Medicine, Kyoto, Japan

4. Center for Innovation in Immunoregulative Technology and Therapeutics, Kyoto University Graduate School of Medicine, Kyoto, Japan

5. $\quad$ NTT Service Evolution Laboratories, Kanagawa, Japan

6. Division of Medical Information Technology \& Administration Planning, Kyoto University Hospital, Kyoto, Japan

\section{Corresponding author:}

Shu Nishiguchi, R.P.T., M.Sc.

Department of Physical Therapy, Human Health Sciences, Kyoto University Graduate School of Medicine, Kyoto, Japan

53 Kawahara-cho, Shogoin, Sakyo-ku, Kyoto 606-8507, Japan

E-mail: nishiguchi.shu.82s@st.kyoto-u.ac.jp, Phone: +81-75-751-3935, Fax: +81-75-751-3909 


\section{Abstract \\ Objectives}

The disease activities of rheumatoid arthritis (RA) tend to fluctuate between visits to doctors, and a self-assessment tool can help patients accommodate to their current status at home. The aim of the present study was to develop a novel modality to assess the disease activity of RA by a smartphone without the need to visit a doctor.

\section{Subjects and Methods}

This study included 65 patients with RA, age $63.1 \pm 11.9$ years. The 28-joint disease activity score (DAS28) was measured for all participants at each clinic visit. The patients assessed their status with the modified health assessment questionnaire (mHAQ), a self-assessed tender joint count (sTJC), and a self-assessed swollen joint count (sSJC) in a smartphone application. The patients' trunk acceleration while walking was also measured with a smartphone application. The peak frequency, auto correlation peak (AC), and coefficient of variance of the acceleration peak intervals were calculated as the gait parameters.

\section{Results}

Univariate analyses showed that the DAS28 was associated with mHAQ, sTJC, sSJC, and AC $(p<0.05)$. In a stepwise linear regression analysis, mHAQ $(\beta=0.264, \mathrm{p}<0.05)$, sTJC $(\beta=0.581, \mathrm{p}<0.001)$, and AC $(\beta=$ $-0.157, \mathrm{p}<0.05)$ were significantly associated with DAS28 in the final model, and the predictive model explained $67 \%$ of the DAS28 variance.

\section{Conclusion}

The results suggest that non-invasive self-assessment of a combination of joint symptoms, limitations of daily activities, and walking ability can adequately predict disease activity of RA with a smartphone application.

\section{Key words}

Rheumatoid arthritis, Disease activity, Smartphone, Self-assessment 


\section{Introduction}

Rheumatoid arthritis (RA) affects approximately 1\% of adults and has been recognized as one of the most serious rheumatologic conditions in the developed world ${ }^{1}$. RA is a progressive inflammatory disease that causes multiple associated joint damages, decline in functional status, and premature mortality ${ }^{2,3}$. Treatment comprises medication to control inflammation and multidisciplinary interventions to reduce symptoms and maximize self-management ${ }^{4}$.

The disease activity of RA fluctuates from day to day and disabilities and reduced activities of daily living are mostly the outcome of deleterious disease activities. To control this deterioration, effective, frequent clinical assessments are necessary. Versions of the disease activity score (DAS/DAS28/DAS28-CRP(4)), which are generally used as valid assessment tools include 4 parameters: tender joint count (TJC), swollen joint count (SJC), C-reactive protein (CRP) level or erythrocyte sedimentation rate (ESR), and patient-assessed disease activity on a visual analog scale (VAS) ${ }^{5,6}$. Since DAS measurement in clinical practice requires a blood sample for CRP or ESR levels, visit to a doctor is necessary. It has not been possible to objectively assess disease activity at home on a daily basis, and it is possible that disease activities undergo change between doctor visits. An objective measurement that can be made easily, daily, and non-invasively at home will greatly help patients with RA.

Smartphones as self-management or rehabilitation tools have recently become widely used for heart failure ${ }^{7}$, diabetes management ${ }^{8}$, and pulmonary rehabilitation ${ }^{9}$. Smartphones have become ubiquitous devices, are now less expensive, and can save large amounts of data and convey these data via both wireless transmission and e-mail. Our previous studies, furthermore, indicated that the smartphone accelerometer has the capacity to measure gait parameters accurately ${ }^{10}$, and disease activity of RA was significantly associated with the gait parameters recorded by the smartphone ${ }^{11}$. Therefore, we hypothesized that patients with RA may also be able to easily self-monitor their daily disease activity at home by using the smartphone.

The aim of the present study was to develop a novel method to assess the disease activity of RA by a smartphone without laboratory tests or the need to visit a doctor. We focused on joint symptoms, activities of daily living (ADL), and gait parameters as daily and non-invasive measurements that predicted disease 
activity.

\section{Patients and Methods}

\section{Patients \& device}

The participants were $67 \mathrm{RA}$ patients (mean age, $63.1 \pm 11.9$ years) who attended the rheumatology outpatient clinic of Kyoto University Hospital. Patients with RA defined by the American College of Rheumatology 1987 or 2010 criteria were included. We excluded patients based on the following exclusion criteria: other musculoskeletal disorders, cognitive disorders, Parkinson's disease, stroke, or unable to walk unassisted over $15 \mathrm{~m}$ using walking aids. Patients with previous surgery in the lower extremities were also excluded. The patients' medications were not changed during the study period.

The smartphone (dimensions: $119 \mathrm{~mm} \times 60 \mathrm{~mm} \times 10.9 \mathrm{~mm}$; weight: $121 \mathrm{~g}$; AQUOS PHONE f SH-13C; Android 2.3; Sharp Co., Osaka, Japan) used in this study included an acceleration sensor, a recording device, and an application for processing the acceleration signals. We also installed an application in the smartphone that allowed patients to measure their daily RA parameters using the application by themselves.

We obtained written informed consent from each participant in accordance with the guidelines approved by the Kyoto University Graduate School of Medicine (approval number E1095) and the Declaration of Human Rights, Helsinki, 1975.

\section{Disease activity of RA}

The DAS28 is usually measured to assess disease activity of RA ${ }^{12}$ at a clinic visit. The DAS28-CRP(4) includes 4 parameters: TJC (out of 28 joints), SJC (out of 28 joints), serum CRP level, and patient’s global assessment of disease status by visual-analogue scale. The DAS28 is generally accepted as a reliable, valid, and responsive measure of disease activity in patients with RA ${ }^{13-15}$. The DAS28-CRP(4) was calculated by rheumatologists.

\section{Measurements with the smartphone application}


The modified health assessment questionnaire (mHAQ), self-assessed TJC (sTJC), and self-assessed SJC (sSJC) were recorded on the smartphone application that we developed. All of these measurements were entered via a touchscreen questionnaire on the smartphone and were measured by participants themselves. The mHAQ is a self-reported measure of physical function. The mHAQ is a widely used and validated tool to quantify functional disability in RA ${ }^{16-19}$. The mHAQ disability index assesses 8 ADL, including dressing and grooming, rising, eating, walking, hygiene, reach, grip, and community activities. The mHAQ is expressed on a scale ranging from 0 to 3 , where $0=$ no disability and $3=$ severe functional disability. The sTJC and sSJC were reported according to 49 (sTJC) or 46 (sSJC) joints used by American College of Rheumatology (ACR) ${ }^{20}$ in a smartphone application (Figure 1).

\section{Gait analysis}

The participants were instructed to walk along a 15-m walkway at their preferred speed. All participants wore their usual walking shoes, avoiding high heels and hard-soled shoes. Trunk linear accelerations were measured by participants themselves with the smartphone as they walked on the walkway. The smartphone was kept adjacent to the L3 spinous process, which is close to where the body's center of mass is believed to be located during quiet standing ${ }^{21}$ using a semi-elastic belt. The accelerometer of the smartphone sampled at $33 \mathrm{~Hz}$. The recorded signals were analyzed by an application developed in the android environment.

The following gait parameters were calculated, according to previous studies: peak frequency (PF) ${ }^{22}$, autocorrelation peak (AC) ${ }^{22,23}$, and coefficient of variance (CV) of the acceleration peak intervals ${ }^{24,25}$. The PF value indicates the gait cycle, which is the time taken for 1 step. The AC value indicates the degree of gait balance, so a higher AC value indicates a greater degree of balance. The CV value indicates the degree of gait variability, i.e., the variability in the elapsed time between the first contacts of 2 consecutive footfalls. To calculate the gait parameters, we used the absolute values of the tri-axial acceleration data to decrease the influence of the measurement terminal posture. Then $a_{t_{1} t_{n}}=a_{t_{1}}, a_{t_{2}}, \ldots, a_{t_{n}}$ denoted the set of all acceleration absolute values acquired from time $t_{1}$ to $t_{n}$, for $t_{1} \leq t_{n}$ and $a_{t}$ and $n$, respectively, denoted the 
acceleration absolute value at time $t$ and the number of all acceleration absolute values acquired from time $t_{1}$ to $t_{n}$. Our previous research indicated that the smartphone with the gait analysis application had the capacity to measure gait parameters with the same accuracy as the conventional tri-axial accelerometer ${ }^{10}$. The details of calculation methods of these gait parameters are described in our previous studies ${ }^{10,11}$.

\section{Statistical analysis}

The relationship between the DAS28-CRP(4) and the measurements that were recorded by patients with the smartphone application was examined using Spearman's correlation coefficient. A multivariate linear regression model using a stepwise method was used to examine which model was the most sensitive potential predictor for the DAS28-CRP(4). Independent variables included the mHAQ, sTJC, sSJC, PF, AC, and CV. Statistical analyses were carried out using the SPSS version 20.0 software package (SPSS, Chicago, IL, USA), with p value of $<0.05$ accepted as significant.

\section{Results}

Of the 67 participants, 2 withdrew from the present study because it was difficult for them to understand the use of the smartphone, and the data of the remaining 65 participants were analyzed accordingly. Of these 65 participants, 18 took disease-modifying anti-rheumatic drugs (DMARDs), 11 took biological drugs, 38 took methotrexate, 18 took steroid drugs, and 15 took non-steroidal anti-inflammatory drugs (NSAIDs). Table 1 shows the demographic and clinical characteristics of study participants. The participants were relatively elderly and had established disease with an average duration of 12.7 years. Despite these population characteristics, the average DAS28-CRP(4) was 2.44, and most of the participants had well controlled disease. Typical acceleration waveforms of gait analysis of patients with RA are shown in Figure 2. The waveform of a patient with relatively slight disease (top panel) is regular and indicates that the patient walks stably. On the other hand, the waveform of a patient with relatively severe disease (bottom panel) is irregular and indicates that the patient walks unstably. 
To determine the association of DAS28-CRP(4) with other measurements, we analyzed Spearman's correlation coefficients. In the correlation analysis, the DAS28-CRP(4) was significantly correlated with mHAQ $(\rho=0.648, \mathrm{p}<0.001), \operatorname{sTJC}(\rho=0.795, \mathrm{p}<0.001), \operatorname{sSJC}(\rho=0.532, \mathrm{p}<0.001)$, and AC $(\rho=-0.309$, $\mathrm{p}=0.024)$

To develop the most sensitive potential predictor of DAS28, a stepwise multiple linear regression analysis was carried out and revealed that the independent variables retained in the final model were sTJC $(\beta=0.581$, $\mathrm{p}<0.001)$, mHAQ $(\beta=0.264, \mathrm{p}=0.013)$, and $\mathrm{AC}(\beta=-0.157, \mathrm{p}=0.043)$, and this model explained $67 \%$ of the DAS28-CRP(4) variance (Table 2). The excluded variables in this model were sSJC, PF, and CV. Therefore, the predictive model of DAS28 was calculated as the following formula using each regression coefficient: predictive model of DAS28 $=2.380+(0.110 \times s T J C)+(0.080 \times m H A Q)+(-1.187 \times A C)$. The correlation between DAS28-CRP(4) and the predictive model of DAS28 is shown in Figure 3, and the predictive model was significantly and strongly correlated with DAS28-CRP(4) $(\rho=0.817, \mathrm{p}<0.001)$.

\section{Discussion}

The results in the present study indicate that non-invasive measurements made using a smartphone can sensitively predict the daily disease activity of RA. Moreover, the predictive model of DAS28 calculated on the basis of linear regression analysis was strongly associated with DAS28-CRP(4).

A hypothesis of the present study was that the daily disease activity of RA could be predicted by non-invasive measurements such as joint symptoms, ADL, and gait parameters. The predictive model by stepwise multiple regression analysis consisted of the sTJC, mHAQ, and AC values. The sTJC was the most strongly associated with DAS28 of the 3 measurements in the predictive model because the TJC as an indicator of a patient's joint symptoms is a part of DAS28 ${ }^{12}$. The TJC of the DAS28 is taken from 28 joints that mainly include the joints of the upper extremities and the knee joints. In the present study, the sTJC was taken from 49 joints including the joints of lower extremities such as the ankle joints, in addition to the joints assessed in the TJC of the DAS28. Therefore, the predictive model reflected the joint symptoms of the whole body to a greater extent than that obtained using only 28 joints. The mHAQ indicates the level of a patient's 
ADL, which is one of the most critical functional parameters for patients with RA ${ }^{16}$. The mHAQ can measure a patient's ADL quickly and be correlated with RA clinical variables ${ }^{4}$. Ideally, it should be measured on a daily basis. The value of AC is known as the degree of gait balance. Previous studies have demonstrated that RA may lead to gait disorders including decreasing walking speed ${ }^{26}$, decreasing hip and knee joint moments ${ }^{27}$, shortening stride length, and increasing double-stance period ${ }^{28}$. Moreover, our previous research indicated that AC was independently associated with RA disease activity ${ }^{11}$. Therefore, the gait parameters have potential as a new index that may be measured daily for patients with RA.

Self-management is an important treatment option for patients with RA, and its use has been investigated previously ${ }^{29}$. Great benefit is observed if self-management programs are maintained for over 8 years ${ }^{30}$, and other studies have demonstrated online self-management systems for patients with RA ${ }^{31}$ and touchscreen questionnaire systems for patient data collection ${ }^{32}$. Smartphones may be useful devices for self-management by patients with RA because of their telecommunication facility, and they are now ubiquitous and have multiple features. With these devices and our smartphone applications, the availability of web-based interventions to support self-management of patients with RA should be further investigated in a study similar to the previous feasibility study for patients with diabetes ${ }^{33}$.

The predictive model in the present study may play an important role for patients with RA in self-management of their disease activity on a daily basis because it offers several benefits. First, the application does not include invasive measurements like blood testing, but daily measurements (joint symptoms, ADL, and gait parameters) that can be made using a smartphone. Second, disease activity can be represented objectively by the gait measurements in the predictive model. Third, medical staff and patients may be able to share information regarding the patient's condition at home in real time using the functions of the smartphone. As a consequence, patients can receive timely advice from their medical providers and seek interventions before acute exacerbation of symptom. In the present study, however, 2 patients withdrew because it was difficult for them to understand the use of the smartphone. This indicates that we should make our application easier to use in future trials.

There were several limitations in the present study that need to be mentioned. First, this study was a pilot 
study and was based on a cross-sectional design, and therefore, we should conduct a longitudinal study in the future to examine temporal transitions of the relationship between disease activity and the predictive model. Second, TJC and SJC in the present study (sTJC and sSJC) were subjectively assessed by the patients themselves. Clinically, on the other hand, these parameters are objectively assessed by physicians. Nonetheless, the results in the present study did indicate that even the sTJC and sSJC can predict a patient's daily disease activity. We should examine this in detail in a future study. Third, the sample size was relatively small. To more sensitively predict the disease activity without the invasive clinical measurements, a large number of patients will be necessary in future studies, considering on validation setting, various races, and multicenter study.

In conclusion, the results suggest that it is possible to sufficiently predict a DAS28 by combining subjective measurements of a patient's joint symptoms, degree of disability, and objective gait balance measurements. This is the first study to predict the DAS28 using non-invasive measurements that may be made daily using a smartphone. The predictor for DAS28 described in the present study may be an acceptable and useful assessment tool of RA disease activity for both patients and medical providers.

\section{Acknowledgements}

We would like to thank all the volunteers for participating in the study.

\section{Conflict of interest statement}

Tetsuya Ura, Akio Shinohara, and Tatsuaki Ito are employees of NTT Service Evolution Laboratories. All other authors have no conflicts of interest to declare. 


\section{References}

1. Alamanos Y, Drosos AA. Epidemiology of adult rheumatoid arthritis. Autoimmun Rev. 2005; 4: 130-136.

2. Murphy D. Lycra working splint for the rheumatoid arthritic hand with MCP ulnar deviation. Aust $J$ Rural Health. 1996;4 : 217-220.

3. Plant MJ, Williams AL, O'Sullivan MM, et al. Relationship between time-integrated C-reactive protein levels and radiologic progression in patients with rheumatoid arthritis. Arthritis Rheum. 2000; 43: 1473-1477.

4. Luqmani R, Hennell S, Estrach C, et al. British Society for Rheumatology and british health professionals in Rheumatology guideline for the management of rheumatoid arthritis (the first two years). Rheumatology (Oxford). 2006; 45: 1167-1169.

5. van der Heijde DM, van 't Hof MA, van Riel PL, et al. Judging disease activity in clinical practice in rheumatoid arthritis: first step in the development of a disease activity score. Ann Rheum Dis. 1990; 49: 916-920.

6. van der Heijde DM, van't Hof MA, van Riel PL, et al. Validity of single variables and composite indices for measuring disease activity in rheumatoid arthritis. Ann Rheum Dis. 1992; 51: 177-181.

7. Seto E, Leonard KJ, Cafazzo JA, et al. Mobile phone-based telemonitoring for heart failure management: a randomized controlled trial. J Med Internet Res. 2012; 14: e31.

8. Holtz B, Lauckner C. Diabetes management via mobile phones: A systematic review. Telemed J E Health. 2012; 18: 175-184.

9. Marshall A, Medvedev O, Antonov A. Use of a smartphone for improved self-management of Pulmonary rehabilitation. Int $J$ Telemed Appl. 2008: 753064.

10. Nishiguchi S, Yamada M, Nagai K, et al. Reliability and validity of gait analysis by android-based smartphone. Telemed J E Health. 2012; 18: 292-296.

11. Yamada M, Aoyama T, Mori S, et al. Objective assessment of abnormal gait in patients with rheumatoid arthritis using a smartphone. Rheumatol Int. 2012; 32 (12): 3869-3874. 
12. Prevoo ML, van 't Hof MA, Kuper HH, et al. Modified disease activity scores that include twenty-eight-joint counts. Development and validation in a prospective longitudinal study of patients with rheumatoid arthritis. Arthritis Rheum. 1995; 38: 44-48.

13. van der Heijde DM, van 't Hof M, van Riel PL, van de Putte LB. Validity of single variables and indices to measure disease activity in rheumatoid arthritis. $J$ Rheumatol. 1993; 20: 538-541.

14. Uhlig T, Kvien TK, Pincus T. Test-retest reliability of disease activity core set measures and indices in rheumatoid arthritis. Ann Rheum Dis. 2009; 68: 972-975.

15. Fransen J, Hauselmann H, Michel BA, et al. Responsiveness of the self-assessed rheumatoid arthritis disease activity index to a flare of disease activity. Arthritis Rheum. 2001; 44: 53-60.

16. Pincus T, Summey JA, Soraci SA, et al. Assessment of patient satisfaction in activities of daily living using a modified Stanford Health Assessment Questionnaire. Arthritis Rheum. 1983; 26: 1346-1353.

17. Bruce B, Fries JF. The Stanford Health Assessment Questionnaire: a review of its history, issues, progress, and documentation. J Rheumatol. 2003; 30: 167-178.

18. Ziebland S, Fitzpatrick R, Jenkinson C, Mowat A. Comparison of two approaches to measuring change in health status in rheumatoid arthritis: the Health Assessment Questionnaire (HAQ) and modified HAQ. Ann Rheum Dis. 1992; 51: 1202-1205.

19. Uhlig T, Haavardsholm EA, Kvien TK. Comparison of the Health Assessment Questionnaire (HAQ) and the modified HAQ (MHAQ) in patients with rheumatoid arthritis. Rheumatology (Oxford). 2006; 45: 454-458.

20. Nishimoto N, Miyasaka N, Yamamoto K, et al. Long-term safety and efficacy of tocilizumab, an anti-IL-6 receptor monoclonal antibody, in monotherapy, in patients with rheumatoid arthritis (the STREAM study): evidence of safety and efficacy in a 5-year extension study. Ann Rheum Dis. 2009; 68: 1580-1584.

21. Moe-Nilssen R, Helbostad JL. Trunk accelerometry as a measure of balance control during quiet standing. Gait Posture. 2002; 16: 60-68.

22. Auvinet B, Berrut G, Touzard C, et al. Reference data for normal subjects obtained with an 
accelerometric device. Gait Posture. 2002; 16: 124-134.

23. Moe-Nilssen R, Helbostad JL. Estimation of gait cycle characteristics by trunk accelerometry. $J$ Biomech. 2004; 37: 121-126.

24. Hausdorff JM, Rios DA, Edelberg HK. Gait variability and fall risk in community-living older adults: a 1-year prospective study. Arch Phys Med Rehabil. 2001; 82: 1050-1056.

25. Beauchet O, Allali G, Annweiler C, et al. Gait variability among healthy adults: low and high stride-to-stride variability are both a reflection of gait stability. Gerontology. 2009; 55: 702-706.

26. Eppeland SG, Myklebust G, Hodt-Billington C, Moe-Nilssen R. Gait patterns in subjects with rheumatoid arthritis cannot be explained by reduced speed alone. Gait Posture. 2009; 29: 499-503.

27. Weiss RJ, Wretenberg P, Stark A, et al. Gait pattern in rheumatoid arthritis. Gait Posture. 2008; 28: 229-234.

28. Laroche D, Pozzo T, Ornetti P, et al. Effects of loss of metatarsophalangeal joint mobility on gait in rheumatoid arthritis patients. Rheumatology (Oxford). 2006; 45: 435-440.

29. Hewlett S, Ambler N, Almeida C, et al. Self-management of fatigue in rheumatoid arthritis: a randomised controlled trial of group cognitive-behavioural therapy. Ann Rheum Dis. 2011; 70: 1060-1067.

30. Barlow J, Turner A, Swaby L, et al. An 8-yr follow-up of arthritis self-management programme participants. Rheumatology (Oxford). 2009; 48: 128-133.

31. Smarr KL, Musser DR, Shigaki CL, et al. Online self-management in rheumatoid arthritis: a patient-centered model application. Telemed $J$ E Health. 2011; 17: 104-110.

32. Newman ED, Lerch V, Jones JB, Stewart W. Touchscreen questionnaire patient data collection in rheumatology practice: development of a highly successful system using process redesign. Arthritis Care Res. 2012; 64: 589-596.

33. Nes AA, van Dulmen S, Eide E, et al. The development and feasibility of a web-based intervention with diaries and situational feedback via smartphone to support self-management in patients with diabetes type 2. Diabetes Res Clin Pract. 2012; 97(3): 385-93. 

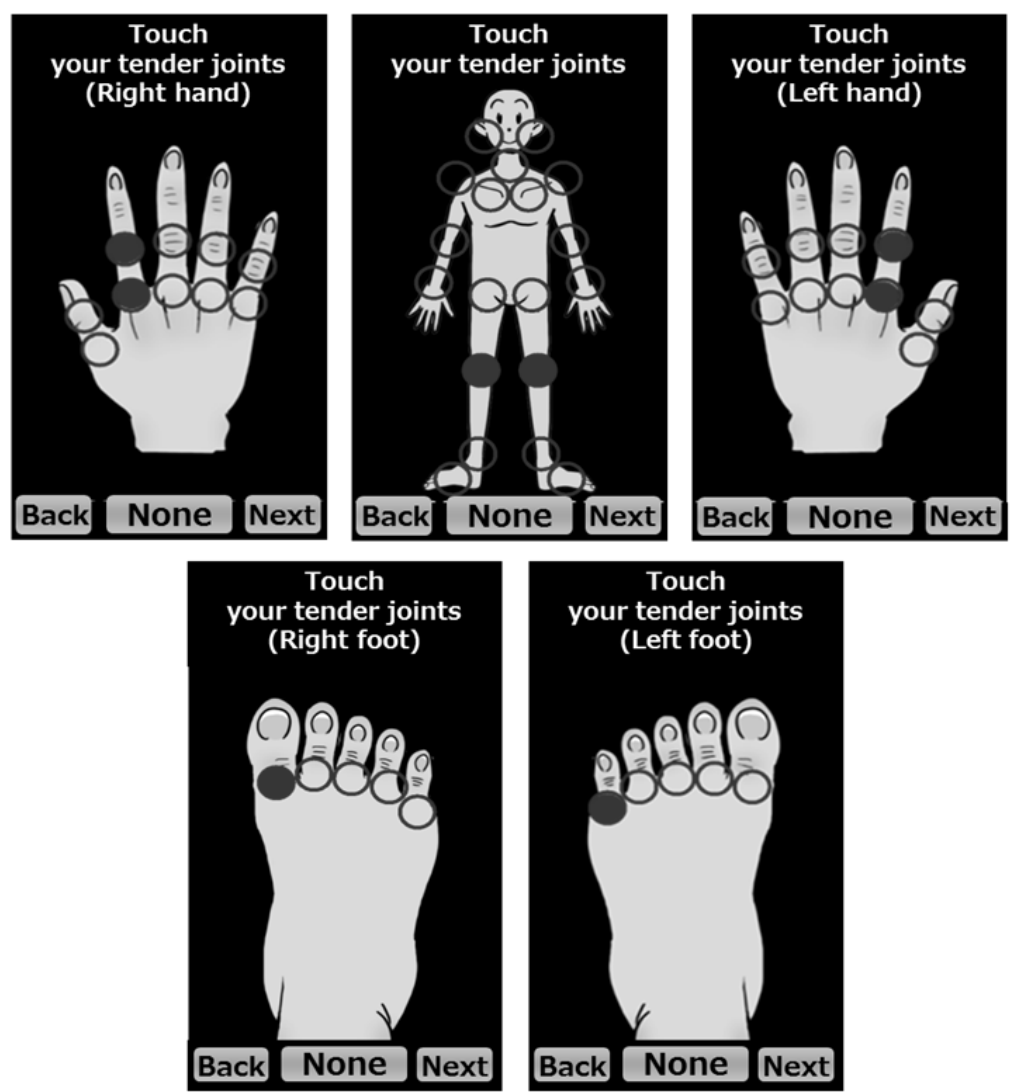

Figure 1. Simulation of the smartphone touchscreen for inputting the number of tender joints and swollen joints (similar screen).

Patients assess their tender joint count and swollen joint count in accordance with the 49 or 46 joints used by the ACR and touch the pertinent joints on the smartphone screen.

The characters of these screens are translated in English. 


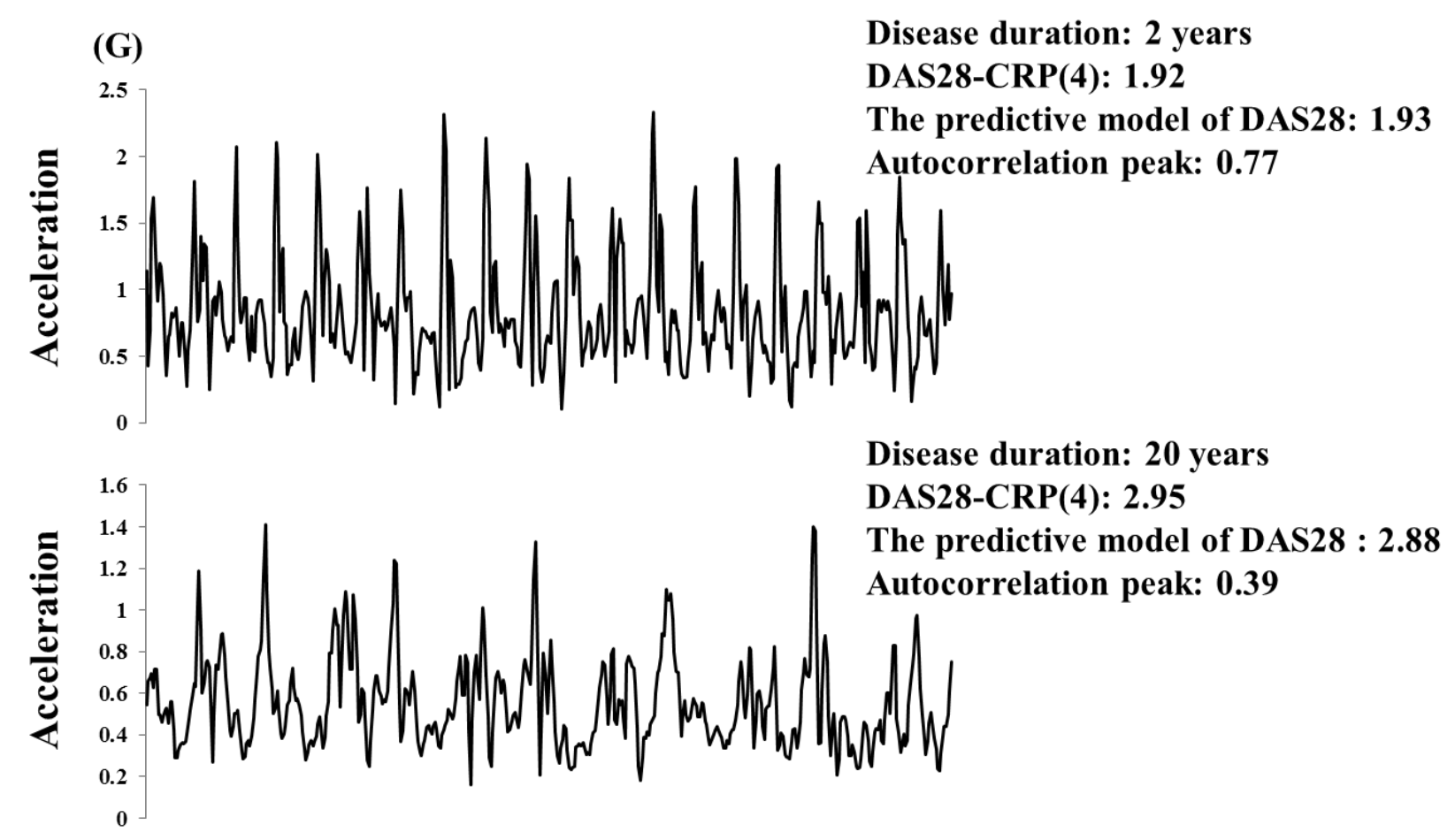

Figure 2. Examples of acceleration waveforms showing patients with slight or severe disease activity.

The waveform of a patient with relatively slight disease (top panel) is regular and indicates that the patient walks stably (DAS28-CRP $(4)=1.92$, predictive model of DAS28 $=1.93$, autocorrelation peak $($ AC $)=0.77$ ).

The waveform of a patient with relatively severe disease (bottom panel) is irregular and indicates that the patient walks unstably (DAS28-CRP(4) $=2.95$, predictive model of DAS28 $=2.88$, AC $=0.39$ ).

DAS: disease activity score. 


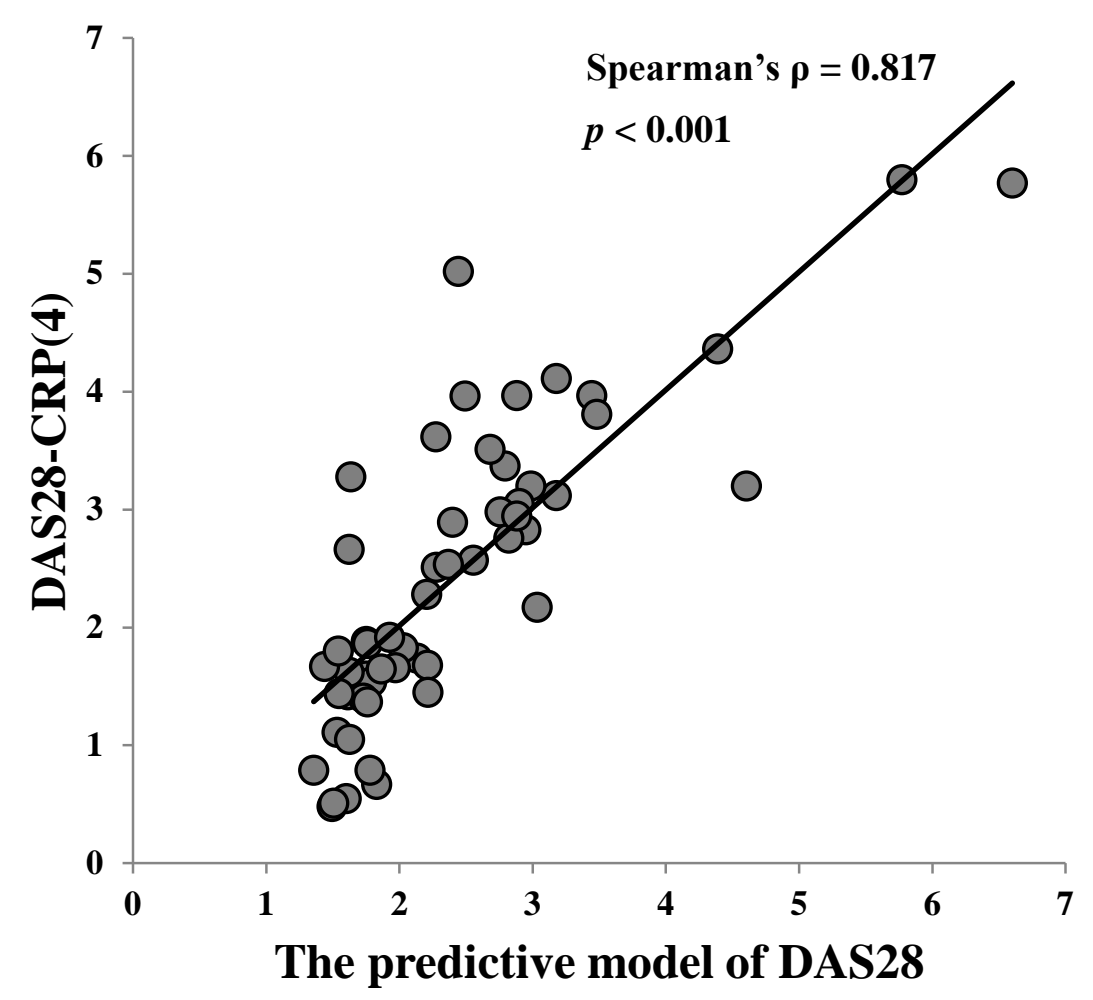

Figure 3. Relationship between DAS28-CRP(4) and the predictive model of DAS28.

DAS28-CRP(4) was significantly and strongly correlated with the predictive model of DAS28, which included sTJC, mHAQ, and AC. 
Table 1. Demographic and clinical characteristics of study participants.

\begin{tabular}{lc}
\hline & Mean \pm SD \\
\hline Age (y) & $63.1 \pm 11.9$ \\
Gender female (n (\%)) & $60(90.9 \%)$ \\
Height (cm) & $154.6 \pm 6.8$ \\
Weight (kg) & $54.6 \pm 9.7$ \\
Disease duration (y) & $12.7 \pm 10.0$ \\
DAS28-CRP(4) (point) & $2.44 \pm 1.28$ \\
mHAQ (point) & $3.62 \pm 4.21$ \\
sTJC (n) & $4.65 \pm 6.68$ \\
sSJC (n) & $3.23 \pm 5.37$ \\
VAS (mm) & $39.1 \pm 26.6$ \\
Gait speed (m/s) & $1.08 \pm 0.29$ \\
PF (Hz) & $2.07 \pm 0.33$ \\
AC & $0.67 \pm 0.18$ \\
CV & $0.17 \pm 0.11$ \\
\hline
\end{tabular}

Abbreviations:

DAS = disease activity score

mHAQ $=$ modified health assessment questionnaire

sTJC = self-assessed tender joint count (0-49)

sSJC $=$ self-assessed swollen joint count (0-46)

VAS= patient's global assessment of disease status on visual-analogue scale

$\mathrm{PF}=$ peak frequency

$\mathrm{AC}=$ autocorrelation peak

$\mathrm{CV}=$ coefficient of variance 
Table 2. Factors associated with disease activity of RA in multiple stepwise regression analysis.

\begin{tabular}{|c|c|c|c|c|c|}
\hline & $\begin{array}{c}\text { Independent } \\
\text { variables }\end{array}$ & $\begin{array}{l}\text { Regression } \\
\text { coefficient }\end{array}$ & $\begin{array}{l}\text { Standard } \\
\text { regression } \\
\text { coefficient }\end{array}$ & $\mathrm{p}$ value & $\mathrm{R}^{2}$ value \\
\hline \multirow[t]{7}{*}{ DAS28-CRP(4) } & sTJC & 0.110 & 0.581 & $<0.001$ & 0.670 \\
\hline & mHAQ & 0.080 & 0.264 & 0.013 & \\
\hline & AC & -1.187 & -0.157 & 0.043 & \\
\hline & sSJC & & - & - & \\
\hline & $\mathrm{PF}$ & & - & - & \\
\hline & $\mathrm{CV}$ & & - & - & \\
\hline & Constant term & 2.380 & & & \\
\hline \multicolumn{6}{|l|}{ Abbreviations: } \\
\hline \multicolumn{6}{|c|}{ DAS = disease activity score } \\
\hline \multicolumn{6}{|c|}{ sTJC = self-assessed tender joint count } \\
\hline \multicolumn{6}{|c|}{ mHAQ = modified health assessment questionnaire } \\
\hline \multicolumn{6}{|c|}{$\mathrm{AC}=$ autocorrelation peak } \\
\hline \multicolumn{6}{|c|}{ sSJC $=$ self-assessed swollen joint count } \\
\hline \multicolumn{6}{|c|}{$\mathrm{PF}=$ peak frequency } \\
\hline $\mathrm{CV}=$ coefficient & rariance & & & & \\
\hline
\end{tabular}

